\title{
Numerical modelling of transient liquid phase bonding and other diffusion controlled phase changes
}

\author{
T. C. ILLINGWORTH*, I. O. GOLOSNOY, V. GERGELY, T. W. CLYNE \\ Department of Materials Science, University of Cambridge, Pembroke Street, Cambridge, \\ CB230Z,UK \\ E-mail: tci20@cam.ac.uk
}

Diffusion in material of inhomogeneous composition can induce phase changes, even at a constant temperature. A transient liquid phase (TLP), in which a liquid layer is formed and subsequently solidifies, is one example of such an isothermal phase change. This phenomenon is exploited industrially in TLP bonding and sintering processes. Successful processing requires an understanding of the behaviour of the transient liquid layer in terms of both diffusion-controlled phase boundary migration and capillarity-driven flow.

In this paper, a numerical model is presented for the simulation of diffusion-controlled dissolution and solidification in one dimension. The width of a liquid layer and time to solidification are studied for various bonding conditions. A novel approach is proposed, which generates results of a high precision even with coarse meshes and high interface velocities. The model is validated using experimental data from a variety of systems, including solid/solid diffusion couples. (C) 2005 Springer Science + Business Media, Inc.

\section{Introduction}

Transient liquid phases (TLPs) are observed in many alloy systems. However, commercial exploitation of the phenomenon is relatively limited. This is partly because current understanding of the process is incomplete, rendering the optimisation of various process variables and design parameters difficult.

Qualitatively, TLPs are easily understood; a full description of the underlying physical processes has been given by MacDonald and Eagar [1]. The essential requirement is that the liquidus temperature of an alloy varies with composition. It is then possible for variation of concentration in an inhomogeneous alloy to cause localised melting at temperatures where the bulk of the material remains solid. If liquid and solid of different compositions are in contact, diffusion will change the concentration profile and can cause an initial widening of the liquid layer, followed by solidification, even during an isothermal heat treatment. Such diffusioncontrolled solidification can be used to bond particles in a powder compact (TLP sintering) or to join larger objects (TLP bonding).

Once solidified, the final composition in the vicinity of a joint can be relatively homogenous. As a result, its properties (such as strength and remelt temperature) can approach those of the parent material. The transient presence of a liquid phase also affords advantages over other joining techniques. Flow under the influence of capillary forces will naturally tend to eliminate any porosity within a bond, without the need to impose any external pressure. In the case of TLP sintering, the liquid phase can additionally promote particle rearrangement, leading to rapid densification of a green body.

The transience of the liquid phase arises due to changes in composition which are a result of diffusion. In the next section, the physical processes which underlie TLPs are examined and a model is developed in which they are simulated.

\section{Modelling}

2.1. Mathematical description of the problem

Diffusion in both liquid and solid is assumed to be governed by Fick's second law (the "Diffusion Equation"),

$$
\frac{\partial c(x, t)}{\partial t}=\frac{\partial}{\partial x}\left(D(c(x, t)) \frac{\partial c(x, t)}{\partial x}\right),
$$

where the composition, $c(x, t)$, is a function of both position $(x)$ and time $(t) . D(c(x, t))$ is the diffusion coefficient of solute in either the liquid or solid phase, which depends on both temperature and concentration. The diffusion Equation 1 has been solved for a range of initial and boundary conditions. However, TLPs have solid and liquid phases which change size over time; this introduces a moving boundary condition, which complicates the analysis. 


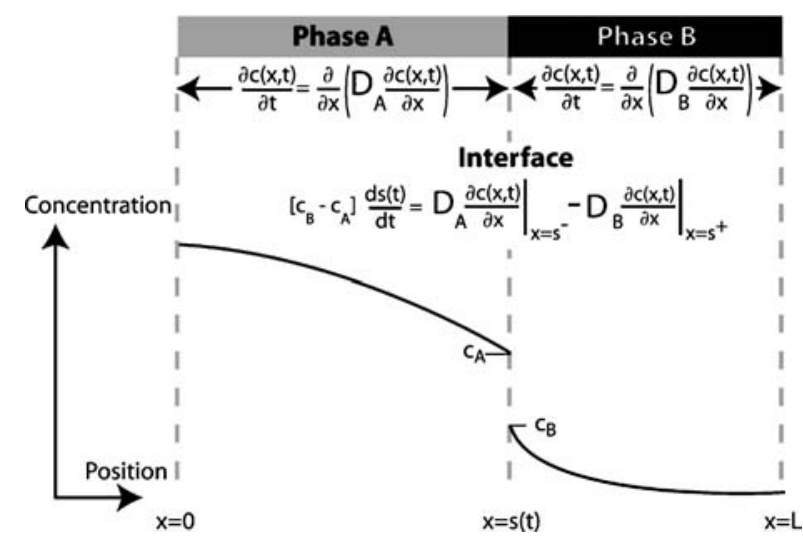

Figure 1 Schematic diagram showing the concentration profile across a TLP bond at one instant in time. Only half of the joint is shown; the other half (from $x=-L$ to $x=0$ ) will be symmetrical.

A one dimensional planar geometry is assumed, as shown in Fig. 1. The variable $s(t)$ is introduced to describe the position of a solid/liquid interface (which varies as a function of time). The moving boundary problem can then be expressed as [2]:

$$
\begin{aligned}
& \frac{\partial c(x, t)}{\partial t}=\frac{\partial}{\partial x}\left(D_{\mathrm{A}}(c(x, t)) \frac{\partial c(x, t)}{\partial x}\right), \quad 0<x<s(t) \\
& \frac{\partial c(x, t)}{\partial t}=\frac{\partial}{\partial x}\left(D_{\mathrm{B}}(c(x, t)) \frac{\partial c(x, t)}{\partial x}\right), \quad s(t)<x<L \\
& \left.D_{\mathrm{A}}(c(x, t)) \frac{\partial c(x, t)}{\partial x}\right|_{x=s(t)^{-}}-\left.D_{\mathrm{B}}(c(x, t)) \frac{\partial c(x, t)}{\partial x}\right|_{x=s(t)^{+}} \\
& =\left[c_{\mathrm{B}}-c_{\mathrm{A}}\right] \frac{\mathrm{d} s(t)}{\mathrm{d} t} . \quad x=s(t)
\end{aligned}
$$

The first equation describes diffusion to the left of the interface, in phase $A$ (liquid in the case of Fig. 1). The second equation refers to diffusion in phase $B$, to the right of the interface (the solid). The third describes the moving boundary condition at the interface. It can be derived by assuming there is local equilibrium there, and by requiring solute conservation. (The notation $c_{\mathrm{A}}$ and $c_{\mathrm{B}}$ is used for the equilibrium liquidus and solidus concentration.) A complete statement of the problem also requires initial conditions, as well as conditions at the fixed boundaries $x=0$ and $x=L$; these are trivial and are omitted for clarity. Any diffusion-controlled isothermal phase change with a planar geometry will obviously be subject to these governing equations, irrespective of whether the phases are solid or liquid. The model developed below can thus be applied to solid state transformations, as well as TLPs.

Systems of partial differential equations with moving boundary conditions (also known as Stefan problems) arise in a variety of modelling situations across the sciences [3]. For certain idealised cases, analytical solutions are available [3-5]. In general, however, numerical solution methods are required. Several viable approaches have been proposed. Unfortunately, Furzeland [6] concludes that the most effective approach to solving a Stefan problem depends on the exact nature of the problem itself. Zhou et al. [7] describe numerical methods formulated specifically to study TLP problems.

\subsection{Fixed spatial discretisation models}

In modelling TLPs, the main difficulty arises in tracking the motion of solid/liquid interfaces. Previously, a fixed discretisation of the domain $x=0$ to $x=L$ has generally been imposed, and a certain concentration is associated with each point after every timestep. The motion of the interface can then be described in a number of ways.

One approach, used by Nakagawa et al. [8] and Cain et al. [9], is to solve the diffusion equations by imposing the requirement that the interface be located at one of the discretisation points. In this case, only a stepwise motion of the interface is permitted. Such a constraint is physically unrealistic. In addition, restrictions on the interface position probably introduce significant errors into the model, as inaccurate approximations of the interface position will directly affect estimates of the fluxes there (and will therefore also affect the predicted interface motion).

More refined models explicitly take account of the interface position and use a discretised form of Equation 4 to predict its motion. Shinmura et al. [10] used an algorithm based on this approach to investigate possible interlayer materials for bonding nickel. Zhou and North [2] also employed this approach. In an attempt to improve the accuracy of their predictions for the interface motion, they additionally proposed the use of a quadratic expression for the concentration profile near the interface, in order to better estimate the fluxes there.

These two models differ slightly in the way that they solve the diffusion Equations 2 and 3: Shinmura et al. [10] use an explicit expression, whereas Zhou and North [2] claim to use an implicit scheme. Certainly, they solve an implicit form of Equations 2 and 3. However, the three Equations 2-4 are interdependentimplicit methods of calculating future concentration profiles will therefore give rise to expressions involving future interface positions as well as future concentrations. Since their method calculates future interface positions by solving (4) explicitly, their scheme is only semi-implicit overall. As a result, there is a limitation on the size of timestep that can be used to generate a solution.

Sinclair et al. [11] also implemented an explicit version of this same algorithm and used it to investigate the solidification of ternary systems. TLP bonding of systems with three components has been investigated in parallel by Campbell and Boettinger [12]. They used generic software, which was developed to describe general diffusion-controlled transformations (not TLP bonding specifically).

It is relatively simple to derive finite difference approximations of the governing Equations 2-4 if a fixed discretisation of space is imposed. Nevertheless, numerical solutions must be calculated carefully. In particular, during timesteps when the interface moves from one element to its neighbour, schemes developed for fixed meshes will not generally conserve solute. Modifications to ensure the conservation of solute are possible, but they increase the complexity of programming. If the interface moves more than one element, solute conservative programming becomes even more difficult. 


\section{PROCEEDINGS OF THE IV INTERNATIONAL CONFERENCE/HIGH TEMPERATURE CAPILLARITY}

Unfortunately, rapid interface motion is typical of the early stages of TLP bonding. Calculations using large timesteps will therefore contain inaccuracies associated with the non-conservation of solute. Eliminating these errors by reducing the step size has the drawback of increasing the computational effort required to solve the problem. The way in which the accuracy of predictions is affected by any particular choice has nowhere been addressed. In comparison, truncation errors are the only source of inaccuracy for conservative discretisation schemes.

\subsection{Use of variable spatial discretisation}

Instead of 'tracking' the motion of a moving boundary across a fixed spatial discretisation, one alternative is to use a mesh which varies in a way that takes account of the moving interface [3]. It is then possible to ensure that the interface position always coincides with a discretisation point without constraining its motion. This approach was used by Tanzilli and Heckel to model solid-state phase transformations as long ago as 1968 [13]. Kajihara and Kikuchi [14] implemented an implicit version of the same model to investigate the behaviour of solid $\gamma / \alpha / \gamma$ diffusion couples in the $\mathrm{Fe}-\mathrm{Cr}-\mathrm{Ni}$ system.

In the present work, a variable space grid is achieved by introducing a wholesale co-ordinate transformation using two new positional variables: $u=\frac{x}{s(t)}$ and $v=\frac{x-s(t)}{L-s(t)}$. These definitions mean that, for any time, the interval $0<x<s(t)$ coincides with $0<u<1$, and that $s(t)<x<L$ coincides with $0<v<1$. Changing co-ordinate system also means that the governing Equations 2-4 must be modified. Writing $p(u, t)$ as the concentration in phase $A$ (which takes the same value as $c(x, t)$ in $0<x<s(t))$ and $q(v, t)$ as the concentration in phase $B$ (corresponding to $c(x, t)$ in $s(t)<x<L)$, Equations 2-4 become [3]:

$$
\begin{aligned}
& s(t)^{2} \frac{\partial p(u, t)}{\partial t}-u s(t) \frac{\mathrm{d} s(t)}{\mathrm{d} t} \frac{\partial p(u, t)}{\partial u} \\
& =\frac{\partial}{\partial u}\left(D_{\mathrm{A}}(p(u, t)) \frac{\partial p(u, t)}{\partial u}\right), \quad 0<u<1 \\
& {[L-s(t)]^{2} \frac{\partial q(v, t)}{\partial t}-(1-v)[L-s(t)] \frac{\mathrm{d} s(t)}{\mathrm{d} t} \frac{\partial q(v, t)}{\partial v}} \\
& =\frac{\partial}{\partial v}\left(D_{\mathrm{B}}(q(v, t)) \frac{\partial q(v, t)}{\partial v}\right), \quad 0<v<1 \\
& \left.\frac{D_{\mathrm{A}}(p(u, t))}{s(t)} \frac{\partial p(u, t)}{\partial u}\right|_{u=1}-\left.\frac{D_{\mathrm{B}}(q(v, t))}{L-s(t)} \frac{\partial q(v, t)}{\partial v}\right|_{v=0} \\
& =\left[c_{\mathrm{B}}-c_{\mathrm{A}}\right] \frac{\mathrm{d} s(t)}{\mathrm{d} t} \cdot u=1 ; \quad v=0
\end{aligned}
$$

In this new co-ordinate system, the interface is fixed at $u=1$ and $v=0$ for all $t$. Fixing the front in this way means that interface motion can be handled more easily. On the other hand, the derivation of a finite difference scheme that conserves solute becomes more involved.

Using the identities $\frac{\partial(p u)}{\partial u}=p+u \frac{\partial p}{\partial u}$ and $\frac{\partial(p s)}{\partial t}=p \frac{d s}{d t}+s \frac{\partial p}{\partial t}$, Equation 5 can be re-written as

$$
\frac{\partial(\mathrm{ps})}{\partial t}=\frac{\mathrm{d} s}{\mathrm{~d} t} \frac{\partial(p u)}{\partial u}+\frac{1}{s} \frac{\partial}{\partial u}\left(D_{\mathrm{A}} \frac{\partial p}{\partial u}\right) . \quad 0<u<1
$$

Similarly, (6) can be expressed as

$$
\begin{aligned}
\frac{\partial(q[L-s])}{\partial t}= & \frac{\mathrm{d} s}{\mathrm{~d} t} \frac{\partial(q(1-v))}{\partial v} \\
& +\frac{1}{L-s} \frac{\partial}{\partial v}\left(D_{\mathrm{B}} \frac{\partial q}{\partial v}\right) . \quad 0<v<1
\end{aligned}
$$

Discretising the space co-ordinate $u$ at $N$ points $\left(u_{0} \ldots u_{N-1}\right)$, writing $u_{i \pm 1 / 2}$ as the position midway between $u_{i}$ and $u_{i \pm 1}$ and introducing the timestep $\delta \mathrm{t}$ such that $t^{j+1}=t^{j}+\delta t$, the finite volume technique [15] is used to integrate the divergent form (8) over one spacestep and one timestep:

$$
\begin{gathered}
\int_{\mathrm{u}_{i-1 / 2}}^{u_{i+1 / 2}} \int_{t^{j}}^{\mathrm{t}^{j+1}} \frac{\partial}{\partial t}\{p s\} \mathrm{d} t \mathrm{~d} u=\int_{\mathrm{t}^{j}}^{\mathrm{t}^{j+1}} \int_{u_{i-1 / 2}}^{u_{i+1 / 2}} \frac{\partial}{\partial u} \\
\times\left\{\frac{\mathrm{d} s}{\mathrm{~d} t} p u+\frac{D_{\mathrm{A}}}{s} \frac{\partial p}{\partial u}\right\} \mathrm{d} u \mathrm{~d} t .0<u<1
\end{gathered}
$$

Introducing $p_{\mathrm{i}}^{\mathrm{j}+\sigma}$ (and $s^{\mathrm{j}+\sigma}$ ) to represent the concentration at $u_{\mathrm{i}}$ (and the interface position) after a proportion $\sigma$ of the timestep has elapsed, Equation 10 leads to the approximation

$$
\begin{aligned}
& \left(p_{i}^{j+1} s^{j+1}-p_{i}^{j} s^{j}\right)\left(u_{i+1 / 2}-u_{i-1 / 2}\right) \\
& =\frac{\delta t}{s^{j+\sigma}}\left(\left(D_{\mathrm{A}}\right)_{i+1 / 2}^{j+\sigma} \frac{p_{i+1}^{j+\sigma}-p_{i}^{j+\sigma}}{u_{i+1}-u_{i}}\right. \\
& \left.\quad-\left(D_{\mathrm{A}}\right)_{i-1 / 2}^{j+\sigma} \frac{p_{i}^{j+\sigma}-p_{i-1}^{j+\sigma}}{u_{i}-u_{i-1}}\right)+\left(s^{j+1}-s^{j}\right) \\
& \quad \times\left(p_{i+1 / 2}^{j+\sigma} u_{i+1 / 2}-p_{i-1 / 2}^{j+\sigma} u_{i-1 / 2}\right),
\end{aligned}
$$

where $\left(D_{\mathrm{A}}\right)_{i \pm 1 / 2}^{j+\sigma}$ correspond to the diffusion coefficients for the concentrations mid-way between discretisation points. In the remainder of this work, diffusion coefficients will be assumed to be independent of concentration. For a fixed bonding temperature, $\left(D_{\mathrm{A}}\right)_{i \pm 1 / 2}^{j+\sigma}$ will be then be constant, taking a value which will be denoted $D_{\mathrm{A}}$.

Setting $i=1 \ldots N-2$, Equation 11 can be used to generate a set of finite difference approximations for the future compositions in phase A. For $i=0$ or $N-1$, finite difference approximations for the relevant boundary conditions must be applied. At the moving interface $(u=1)$, local equilibrium requires that $p_{N-1}^{j+1}=c_{A}$. At the fixed boundary $(u=0)$, standard techniques [15, 16] can be used to modify (11) so as to ensure zero flux. Doing so gives rise to the finite difference expression

$$
\begin{aligned}
& \left(p_{0}^{j+1} s^{j+1}-p_{0}^{j} s^{j}\right)\left(u_{1 / 2}\right) \\
& \quad=\frac{D_{\mathrm{A}} \delta t}{s^{j+\sigma}}\left(\frac{p_{1}^{j+\sigma}-p_{0}^{j+\sigma}}{u_{1}}\right)+\left(s^{j+1}-s^{j}\right)\left(p_{1 / 2}^{j+\sigma} u_{1 / 2}\right) .
\end{aligned}
$$


Similarly, integration can be used to generate finite difference expressions for future compositions in phase B from Equation 9:

$$
\begin{aligned}
& \left(q_{i}^{j+1}\left(L-s^{j+1}\right)-q_{i}^{j}\left(L-s^{j}\right)\right)\left(v_{i+1 / 2}-v_{i-1 / 2}\right) \\
& =\frac{\delta t}{L-s^{j+\sigma}}\left(D_{\mathrm{B}} \frac{q_{i+1}^{j+\sigma}-q_{i}^{j+\sigma}}{v_{i+1}-v_{i}}-D_{\mathrm{B}} \frac{q_{i}^{j+\sigma}-q_{i-1}^{j+\sigma}}{v_{i}-v_{i-1}}\right) \\
& \quad+\left(s^{j+1}-s^{j}\right)\left(q_{i+1 / 2}^{j+\sigma}\left(1-v_{i+1 / 2}\right)\right. \\
& \left.\quad-q_{i-1 / 2}^{j+\sigma}\left(1-v_{i-1 / 2}\right)\right) .
\end{aligned}
$$

Appropriate boundary conditions are easily derived in the same way as for phase A.

It remains to discretise Equation 7, which describes the motion of the interface and which follows from the requirement that solute be conserved there. A finite difference form can be derived in the same way. If $v$ is discretised at $M-1$ points, the total amount of solute in the system at time $t^{j+1}$ is

$$
\begin{aligned}
s^{j+1} & {\left[p_{0}^{j+1} \frac{u_{1}-u_{0}}{2}+\sum_{i=1}^{N-2} p_{i}^{j+1} \frac{u_{i+1}-u_{i-1}}{2}\right.} \\
& \left.+p_{\mathrm{N}-1}^{j+1} \frac{u_{\mathrm{N}}-u_{\mathrm{N}-1}}{2}\right]+\left(L-s^{j+1}\right)\left[q_{0}^{j+1} \frac{v_{1}-v_{0}}{2}\right. \\
& \left.+\sum_{i=1}^{\mathrm{M}-2} q_{i}^{j+1} \frac{v_{i+1}-v_{i-1}}{2}+q_{\mathrm{M}-1}^{j+1} \frac{v_{\mathrm{M}}-v_{\mathrm{M}-1}}{2}\right]
\end{aligned}
$$

A similar expression gives the total amount of solute at time $t^{j}$. For the model to be conservative, the difference between these two values must be zero. Subtracting one from the other gives rise to terms such as $\frac{u_{i+1}-u_{i-1}}{2}\left[s^{j+1} p_{i}^{j+1}-s^{j} p_{i}^{j}\right]$, for which alternative forms have been derived (Equations 11 and 12). Massive cancellation upon substitution leaves only

$$
\begin{gathered}
\frac{D_{\mathrm{B}} \delta t}{L-s^{j+\sigma}}\left(\frac{q_{1}^{j+\sigma}-c_{\mathrm{B}}}{v_{1}}\right)-\frac{D_{\mathrm{A}} \delta t}{s^{j+\sigma}} \frac{c_{\mathrm{A}}-p_{\mathrm{N}-2}^{j+\sigma}}{1-u_{\mathrm{N}-2}} \\
=\left(s^{j+1}-s^{j}\right)\left[\frac{1+u_{\mathrm{N}-2}}{2} p_{\mathrm{N}-1-1 / 2}^{j+\sigma}+\frac{1-u_{\mathrm{N}-2}}{2} c_{\mathrm{A}}\right. \\
\left.\quad-\left(1-\frac{v_{1}}{2}\right) q_{1 / 2}^{j+\sigma}-\frac{v_{1}}{2} c_{\mathrm{B}}\right] .
\end{gathered}
$$

Equations 11-13 form a finite difference formulation of the problem described analytically in Equations 57. The way in which they were derived has ensured that they conserve solute. Appropriate approximations for terms at intermediate times $(j+\sigma)$ and intermediate positions $(i \pm 1 / 2)$ will be considered presently, along with efficient methods of solving the resulting set of equations.

\subsection{Implementation}

The problems relating to diffusion ((11) and (12)) and interface motion (13) are interdependent: the diffusion equations involve the future interface position; con- versely, for all implicit schemes $(\sigma \neq 0)$, the interface equation involves terms which depend on future concentrations. The concentration profiles and the interface motion therefore form a strongly coupled problem. It follows that the equations cannot be solved independently; instead, they must be solved simultaneously.

Since the system of simultaneous equations is nonlinear, an iterative solution method must be used. This is potentially very demanding in terms of computing time. But careful analysis can significantly improve the efficiency of the algorithm.

Firstly, note that it is trivial to solve Equation 13 for $s^{j+1}$ if estimates are available for $s^{j+\sigma}$ and concentration profiles $p_{i}^{j+\sigma}$ and $q_{i}^{j+\sigma}$ (from which intermediate concentrations $p_{i \pm 1 / 2}^{j+\sigma}$ and $q_{i \pm 1 / 2}^{j+\sigma}$ can be determined). Once $s^{j+1}$ has been calculated, it is easy to find future concentrations $p_{i}^{j+1}$ : assuming that terms such as $p_{i \pm 1 / 2}^{j+\sigma}$ can be approximated sufficiently well using only the concentrations of neighbouring points at the same time (i.e. $p_{i-1}^{j+\sigma}, p_{i}^{j+\sigma}$ and $p_{i+1}^{j+\sigma}$ ), Equation 11 is reduced to a tridigonal form, which can be inverted cheaply. Concentrations in phase B can also be calculated by inverting a tridiagonal matrix which follows from (12).

Having decoupled and linearised the problem in this way, implementation is simple. The results discussed in the following section have been generated by an algorithm which, at each timestep, executes the following procedure:

(1) Take $s^{j}, p_{i}^{j}$ and $q_{i}^{j}$ as initial values for $s^{j+\sigma}, p_{i}^{j+\sigma}$ and $q_{i}^{j+\sigma}$ and calculate intermediate concentration profiles $p_{i+1 / 2}^{j+\sigma}$ and $q_{i+1}^{j+\sigma}$.

(2) Calculate the future interface position, $s^{j+1}$ using (13).

(3) Using this value of $s^{j+1}$, update the estimate for $s^{j+\sigma}$.

(4) Calculate the future interface positions, $p_{i}^{j+1}$ and $q_{i}^{j+1}$ for all $i$ using (11), (12) and the boundary conditions.

(5) Using these values for $p_{i}^{j+1}$ and $q_{i}^{j+1}$, update the estimates for $p_{i}^{j+\sigma}$ and $q_{i+\sigma}^{j+\sigma}$ and calculate intermediate concentration profiles $p_{i+1 / 2}^{j+\sigma}$ and $q_{i+1 / 2}^{j+\sigma}$.

Steps (2)-(5) are then repeated until successive estimates of the interface position $s^{j+1}$ differ by less than some fixed tolerance. If successive refinements do indeed converge to some fixed value, this will correspond to a solution of the implicit set of discretised equations.

It is well known $[16,17]$ that the Crank-Nicolson scheme $(\sigma=1 / 2)$ gives an error that is proportional to $\delta t^{2}$. This would seem preferable the fully implicit scheme $(\sigma=1)$, which generates errors proportional to $\delta t$. However, if the Crank-Nicolson scheme is used to discretise discontinuous composition profiles, unphysical oscillations will be predicted close to the discontinuity unless the timestep is of the order of $\frac{h^{2}}{\max \left(D_{A}, D_{B}\right)}$ (where $h$ is the spacestep) [16]. On the other hand, fully implicit schemes predict monotonic concentration profiles, no matter what timestep 
is chosen [15-17]. Since discontinuous concentration profiles and large diffusion coefficients are inherent to TLPs, attention will be limited to fully implicit schemes.

Monotonicity concerns must also be taken into account when considering possible approximations for the intermediate concentrations $p_{i \pm 1 / 2}^{j+\sigma}$ and $q_{i \pm 1 / 2}^{j+\sigma}$. It is known that second-order centre-difference schemes can produce non-monotonic (oscillating) solutions [15, 17]. In the case of the current problem, trials have indicated that such approximations do indeed generate such unphysical predictions for the TLP problem. Instead, the following fully implicit up/down-wind approximations are used:

- For a positive velocity $\left(s^{j+1}>s^{j}\right)$ :

$$
\begin{array}{ll}
p_{i+1 / 2}^{j+\sigma}=p_{i+1}^{j+1}, & p_{i-1 / 2}^{j+\sigma}=p_{i}^{j+1} \quad \text { and } \\
q_{i+1 / 2}^{j+\sigma}=q_{i+1}^{j+1}, & q_{i-1 / 2}^{j+\sigma}=q_{i}^{j+1} ;
\end{array}
$$

- For a negative velocity $\left(s^{j+1}<s^{j}\right)$ :

$$
\begin{array}{ll}
p_{i+1 / 2}^{j+\sigma}=p_{i}^{j+1}, & p_{i-1 / 2}^{j+\sigma}=p_{i-1}^{j+1} \quad \text { and } \\
q_{i+1 / 2}^{j+\sigma}=q_{i}^{j+1}, & q_{i-1 / 2}^{j+\sigma}=q_{i-1}^{j+1} .
\end{array}
$$

Computer code implementing an algorithm using these approximations has been prepared. Experiments have shown that predictions using these first order approximations for the spatial and temporal variation of composition generate smooth, monotonic profiles. In addition, successive estimates of interface positions at each timestep do indeed converge to fixed values, indicating that linearising and decoupling the problem is a suitable method of finding its solution in an efficient way. Results from the model are presented in the next section.

\section{Validation, results and discussion}

A variety of systems have been investigated, and results agree well with experimental data. In Fig. 2, the half width of the liquid layer is plotted as a function of time for a Ni-P interlayer between pure Ni plates. Following Zhou and North [2], a constant molar volume is assumed (irrespective of phase and composition) ${ }^{1}$. Experimental data and model predictions from the same source are also presented in Fig. 2, for comparison with the present work.

The behaviour of the transient liquid phase predicted by both models is in accordance with our qualitative understanding of the process. The output is also in approximate quantitative agreement with experimental data, even if very few discretisation points are used.

Both models assume that the number of atoms in any given volume does not vary with composition. Since phosphorous is an interstitial element in solid nickel, this assumption is not strictly correct. However, the

\footnotetext{
${ }^{1}$ This assumption will be made in all subsequent calculations.
}

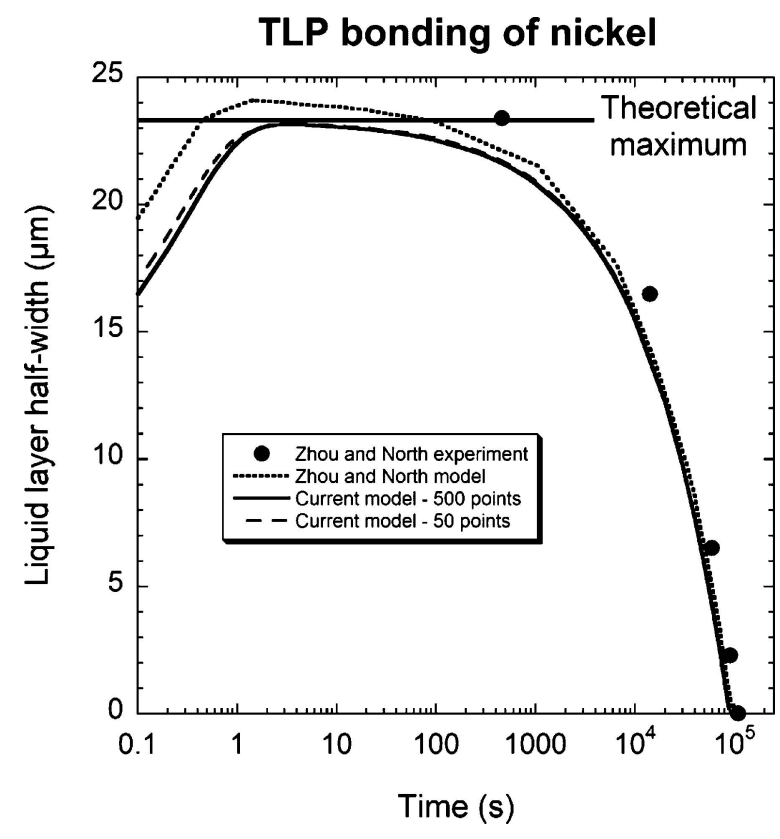

Figure 2 Predicted variation of liquid half-layer thickness with time as $\mathrm{Ni}-\mathrm{P}$ liquid solidifies between two sheets of pure Ni. Experimental data and numerical predictions of Zhou and North [2] are compared with output from the current model, using the same geometry and the same diffusion coefficient for phosphorus in solid or liquid nickel $\left(1.8 \times 10^{-7}\right.$ or $5 \times 10^{-6} \mathrm{~cm}^{2} \mathrm{~s}^{-1}$ respectively). The 'theoretical maximum liquid layer thickness' is calculated by neglecting fluid flow and diffusion in the solid and assuming a constant molar volume.

very low solidus concentration at the bonding temperature $(0.17$ at $\%)$ means that errors arising due to this simplification are probably not significant. Assuming a constant molar volume permits the theoretical maximum liquid layer thickness to be calculated - the thickness at which liquid is diluted to the equilibrium concentration, without any diffusion of solute into the solid. For $12.5 \mu \mathrm{m}$ of Ni-19 at\% $P$ dissolving pure Ni until it reaches a concentration of 10.2 at $\% P$ (the liquidus concentration at the bonding temperature), the theoretical maximum liquid width is $23.2 \mu \mathrm{m}$. The predictions of Zhou and North [2] exceed this value, indicating that their simulation does not conserve solute. The predictions of the present model do not exceed the theoretical maximum, which is consistent with the fact that it does conserve solute.

One experimental datum is also greater than the 'theoretical maximum'. This may be because the assumption of constant molar volume in the liquid is incorrect. Alternatively, liquid flow during experiments may have affected the thickness of the liquid layer as well as diffusion.

Difficulties associated with eliminating liquid flow mean that experimental data for the width of TLPs that are strictly controlled by diffusion are sparse [1]. If neither phase in a diffusion couple is fluid, flow is not a problem. Data for two-phase diffusion-controlled phase transformations in the solid state are more readily available; since these represent mathematically identical problems, this data can be used to further test the model. Heckel et al. [18] investigated the diffusion of $\mathrm{Zn}$ in $\alpha-\beta$ brass diffusion couples. Their data tracking interface positions as a function of time are presented 


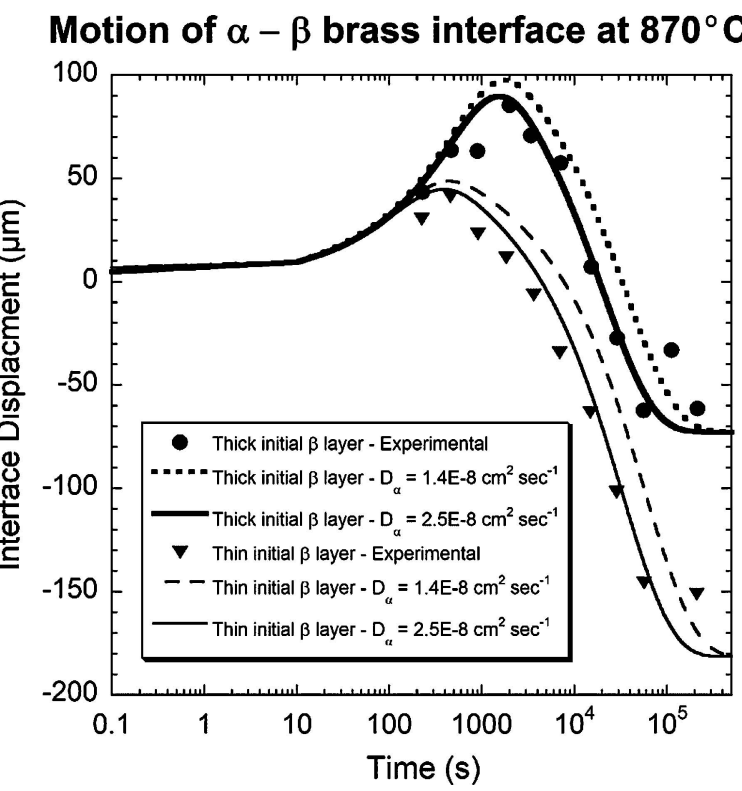

Figure 3 Experimental data and model predictions for the growth and subsequent shrinkage of $\beta$-brass in contact with $\alpha$-brass at $870^{\circ} \mathrm{C}$. Experimental data are from Heckel et al. [18], who used a constant thickness of $\alpha$-brass $(749 \mu \mathrm{m})$ and two different thicknesses of $\beta$-brass (381 $\mu \mathrm{m}$ and $762 \mu \mathrm{m}$ ) to investigate the motion of a phase boundary in this system. For each initial geometry, two corresponding predictions are plotted - the value of the diffusion coefficient of $\mathrm{Zn}$ in $\alpha$-brass is unclear (see text).

in Fig. 3 for two different initial thicknesses of $\beta$ brass (381 and $762 \mu \mathrm{m}$ ).

There is some disagreement in the literature regarding the frequency factors $(A)$ and activation energies $(Q)$ for the diffusion of zinc in Zn-rich $\alpha$ brass at $870^{\circ} \mathrm{C}$. Simulations were completed using two different diffusion coefficients, calculated using the published reference values [19]: either $A=0.016 \mathrm{~cm}^{2} \mathrm{~s}^{-1}$ and $Q=124.5 \mathrm{~kJ} \mathrm{~mol}^{-1}$; or $A=1.7 \mathrm{~cm}^{2} \mathrm{~s}^{-1}$ and $Q=172.9 \mathrm{~kJ} \mathrm{~mol}^{-1}$. At a temperature of $870^{\circ} \mathrm{C}$, these values correspond to diffusion coefficients $D_{\alpha}=$ $1.4 \times 10^{-8} \mathrm{~cm}^{2} \mathrm{~s}^{-1}$ and $D_{\alpha}=2.5 \times 10^{-8} \mathrm{~cm}^{2} \mathrm{~s}^{-1}$. In the $\beta$ phase, the diffusion coefficient was calculated to be $1.4 \times 10^{-6} \mathrm{~cm}^{2} \mathrm{~s}^{-1}$ using the undisputed reference values for $A$ and $Q$.

The simulations using either value of $D_{\alpha}$ qualitatively match the data, and there is close quantitative agreement with the second set of calculations. The differences between the model predictions indicate that user-specified diffusion coefficients have a significant effect on output, as expected. Clearly, correct diffusion coefficients are required for accurate predictions. The use of concentration-dependent diffusion coefficients might also improve the accuracy of the model. However, on account of the paucity of data corresponding to the concentration-dependency of diffusion coefficients in most systems, such an extension has not been attempted.

\section{Conclusions}

A model has been developed to describe isothermal phase changes that are controlled by the diffusion of matter under the assumptions that
(1) There is local equilibrium at the interface;

(2) Diffusion can be described by Fick's second law;

(3) The molar volume in each phase is constant.

The implementation considered in the present work models a two-phase diffusion couple in one dimension (describing a planar geometry). Only binary systems have been considered in the present work, and the diffusion coefficient in each phase is assumed to be independent of composition. None of these conditions are requirements of the method: extensions to cover many phases in higher dimensions, or several components and variable diffusion coefficients are possible.

The numerical scheme requires a system of coupled non-linear equations to be solved. The implementation described above is fully implicit and decouples the problem into a set of linear equations which are solved iteratively. Calculations have been found to converge to accurate solutions, even with large timesteps. Large spacesteps can also be used with this method, as can non-uniform meshes.

Validation of the model has been possible using experimental data from various systems, including TLP and solid-state diffusion couples. Agreement between model and observation is good, even for relatively large steps in space and time. In contrast with previous models of diffusion-controlled phase changes, the scheme also conserves solute. It has first order accuracy in space and time.

The model has been implemented in the $\mathrm{C}$ programming language; optimising the efficiency of the code means that calculations can be completed very rapidly. The code is freely available to download from the Materials Algorithm Project web-site: http://www.msm. cam.ac.uk/MAP

\section{References}

1. W. MACDONALD and T. W. EAGAR, Ann. Rev. Mat. Sci. 22 (1992) 23.

2. Y. ZHOU and T. H. NORTH, Modelling Simul. Mater. Sci. Eng. 1(4) (1993) 505.

3. J. Crank, Free and Moving Boundary Problems (Clarendon Press, Oxford, 1984).

4. Y. ZHOU, J. Mat. Sci. Let., 20(9) (2001) 841.

5. S. LIU, D. L. OLSON, G. P. MARTIN and G. R. EDW A RD S, Welding J. 70(8) (1991) S207.

6. R. M. Furzeland, J. Inst. Maths Appl. 26 (1980) 411.

7. Y. ZHOU, W. F. GALE and T. H. NORTH, Int. Mat. Rev. 40 (1995) 181.

8. H. NAKAGAWA, C. H. LEE and T. H. NORTH,Met. Trans. A 22(2) (1991) 543.

9. S. R. CAIN, J. R. WILCOX and R. VENKATRAMAN, Acta Mat. 45(2) (1997) 701.

10. T. ShinMURA, K. OHSASA and T. NARITA, Mat. Trans. JIM 42(2) (2001) 292.

11. C. W. SinClair, G. R. PURDY and J. E. MORRAL, Met. Mat. Trans. A 31(4) (2000) 1187.

12. C. E. CAMPBELL and W. J. BOETTINGER, ibid. 31(11) (2000) 2835.

13. R. A. TANZILli and R. W. HECKEL, Trans A.I.M.E. 242 (1968) 2312.

14. M. KAJIHARA and M. KIKUCHI, ActaMet.Mat., 41(7) (1993) 2045.

15. M. RAPPAZ, M. BELlet and M. DEVILle, "Numerical Modeling in Materials Science and Engineering," (Springer 
Series in Computational Mathematics, Springer-Verlag, Berlin, 2003).

16. A. A. SAMARSKII and P. N. VABISHEVICH, "Computational heat transfer. V.1. Mathematical Modelling" (Chichester, Wiley, 1995).

17. J. C. TANNEHILl, D. A. ANDERSON and R. H. PLETCHER, "Computational Fluid Mechanics and Heat Transfer," 2nd ed. (Taylor \& Francis, London, 1997).
18. R. W. HECKEL, A. J. HICKL, R. J. ZAEHRING and R. A. TANZILLI, Met. Trans. 3 (1972) 2565.

19. C. J. SMITHELL, "Smithell's Metals Reference Book," 7th ed. (Butterworth, London, 1993).

Received 31 March

and accepted 18 July 2004 